\title{
种群分布格局的多尺度分析
}

\author{
王本洋 余世孝* \\ (中山大学生命科学学院,广州 510275)
}

摘 要 种群分布格局的分析对于了解种群空间分布规律以及种内与种间关系具有重要的意义。最近邻体分析 方法 (Nearest neighbor analysis, NNA) 作为种群空间分布格局的重要分析方法, 仅局限于种群格局的单尺度分析。改 进 NNA 方法以应用于种群格局的多尺度分析, 将有助于解决种群格局的尺度依赖性。该文在前人研究的基础上提 出扩展最近邻体分析方法 (Extended nearest neighbor analysis, ENNA), 也即在传统 Clark-Evans 指数公式的基础上增加 一个距离尺度参数 $d(\mathrm{~m})$, 并定义其所对应的 Clark-Evans 指数 $C E(d)$ 的计算公式及其相应的显著性检验计算公式 $(u(d))$ 分别为: $C E(d)=r_{d \mathrm{~A}} / r_{d \mathrm{E}}=\left(\frac{1}{N_{d}} \sum_{i=1}^{N_{d}} r_{d i}\right) /\left(0.5 \sqrt{A_{d} / N_{d}}+0.0514 P_{d} / N_{d}+0.041 P_{d} / N_{d}{ }^{3 / 2}\right)$ 和 $u(d)=\left(r_{d \mathrm{~A}}-\right.$ $\left.r_{d \mathrm{E}}\right) / \sigma_{d}$, 在距离尺度 $d(\mathrm{~m})$ 范围内, 参数 $r_{d \mathrm{~A}}$ 指样地内各个体与其最近邻体间距离的平均值 $(\mathrm{m}) 、 r_{d \mathrm{E}}$ 指相同环境中个 体呈随机状态时最近邻体距离的平均值 $(\mathrm{m}) 、 N_{d}$ 为样地内个体总数、 $r_{d i}$ 为第 $i$ 个个体与其最近邻体间的距离 $(\mathrm{m})$ 、 $A_{d}$ 为样地面积 $\left(\mathrm{m}^{2}\right) 、 P_{d}$ 为样地周长 $(\mathrm{m})$ 和 $\sigma_{d}$ 代表标准差。ENNA 尺度变换采用与分形理论中计算沙盒维数相类 似的过程, 而格局类型判断的标准与传统最近邻体分析方法相同。传统最近邻体分析结果是 ENNA 中距离尺度 $d$ 取最大值 $d_{\text {max }}$ 时的一个特例。以广东省黑石顶自然保护区针阔叶混交林中的马尾松 ( Pinus massoniana)、黄牛奶树 (Symplocos laurina)、水栗 (Castanopsis nigrescens)、鼠刺( Itea chinensis) 和桃金娘 (Rhodomyrtus tomentosa) 等 5 个代表性种 群为例, 在地理信息系统软件 ArcView GIS 技术平台上进行的实例研究显示, 5 个种群均表现出不同程度的尺度相 关性。由此表明, 该文提出的新方法 ENNA 能够检测出种群空间分布格局的尺度依赖性, 获得关于种群空间分布 格局的多尺度信息, 是进行种群空间格局多尺度分析的有效方法。

关键词 扩展最近邻体分析法 种群 空间分布格局 多尺度分析 GIS

\section{MULTI-SCALE ANALYSES OF POPULATION DISTRIBUTION PATTERNS}

\author{
WANG Ben-Yang and YU Shi-Xiao* \\ ( School of Life Sciences, Sun Yat-sen University, Guangzhou 510275, China)
}

\begin{abstract}
The analyses of spatial distribution patterns of plant populations are useful for understanding pattern types and intra/inter-specific relationships. One of the most frequently employed methods in detecting spatial distribution patterns of populations is the nearest neighbor analysis proposed by Clark and Evans in 1954. This method has been highly successful for analyzing spatial patterns at a single scale but is rarely used for analyzing distribution patterns at multiple scales. We present the extended nearest neighbor analysis (ENNA) in this paper to solve the scale-dependent problem associated with the traditional method of nearest neighbor analysis. The Clark-Evans index was modified by using a distance scale parameter $d(\mathrm{~m})$, described in the following equation: $C E(d)=r_{d \mathrm{~A}} / r_{d \mathrm{E}}=\left(\frac{1}{N_{d}} \sum_{i=1}^{N_{d}} r_{d i}\right) /\left(0.5 \sqrt{A_{d} / N_{d}}+0.0514 P_{d} / N_{d}+0.041 P_{d} / N_{d}^{3 / 2}\right)$. Accordingly, the equation for testing the calculated $C E$ index values against the significant deviation from 1 was changed into $u(d)=\left(r_{d \mathrm{~A}}-r_{d \mathrm{E}}\right) / \sigma_{d}$, where the parameters, $r_{d \mathrm{~A}}, r_{d \mathrm{E}}, N_{d}, r_{d i}, A_{d}, P_{d}, \sigma_{d}$, refer to the mean distance between an individual and its nearest neighbor $(\mathrm{m})$, the expected mean distance of the individuals of a population randomly scattered $(\mathrm{m})$, the number of individuals in the current sample plot, distance between individual $i$ and its nearest neighbor $(\mathrm{m})$, surface of the current sample plot $\left(\mathrm{m}^{2}\right)$, circumference of the current sample plot $(\mathrm{m})$, and the standard deviation, respectively. The procedure of scaling transformation in this approach was similar to that of the sandbox experiment in fractal theory, and the rule for detecting the pattern type was the same as that in the traditional nearest neighbor analysis. The traditional nearest neighbor analysis is a special case for the extended nearest neighbor analysis in which the minimum value of the distance scale
\end{abstract}


parameter $(d)$ is used. An example using the data from a needle and broad-leaved mixed forest community at Heishiding Nature Reserve, Guangdong Province was presented to explain the procedure. Five typical plant populations of this community, Pinus massoniana, Symplocos laurina, Castanopsis nigrescens, Itea chinensis and Rhodomyrtus tomentosa, were chosen for the multi-scale analysis of spatial distribution patterns. The results showed that spatial patterns of all five populations were scale-dependent with varying degrees of intensity. The Pinus massoniana population was randomly distributed at most scales examined, which may have been caused by the random self-thinning process in the population. The population of Itea chinensis was clumped at all scales examined. A simulation with the aid of geographic information system (GIS) also revealed that the distribution patterns of Symplocos laurina, Castanopsis nigrescens, Itea chinensis and Rhodomyrtus tomentosa were mainly clumped or random with an increase of distance scale. These results demonstrated that the ENNA method presented in this paper could be used for multi-scale analysis of spatial distribution patterns of plant populations that could not be solved using the traditional nearest neighbor analysis.

Key words Extended nearest neighbor analysis (ENNA), Spatial distribution patterns, Multi-scale analysis, GIS

种群空间分布格局是指特定时间内, 群落中某 一种群的个体在空间的分布状况。种群分布格局的 分析是研究种群特征、种内和种间关系以及种群与 环境关系的重要手段, 一直是生态学中研究热点之 一。按照种群内个体的聚集程度和方式, 种群分布 格局一般可分为随机分布、均匀分布和集群分布等 3 种类型, 其确定方法主要包括以频度/密度为基础 的样方法和以距离为基础的无样方法两大类 (徐化 成等, 1994; 戴小华和余世孝, 2003)。距离分析方法 以个体与个体之间的距离为原始数据获取分析指 标, 用统记检验的方法考察实际值与理论期望值之 间的差异显著性, 进而判断种群的空间分布类型。

最近邻体分析 ( Nearest neighbor analysis, NAA) 属于无样方法之一, 它最早由 Clark 和 Evans (1954) 提出, 其基本理论是通过比较样地内种群的实际分 布与假设的随机分布之间的差异, 借以作出种群空 间分布类型的判断。它是分析种群空间分布格局的 主要方法之一( Moeur, 1993), 在生态学上得到了广 泛的应用 (Füldner, 1995; 王峥峰等, 1998; Kint et al., 2000; 戴小华和余世孝,2003)。由于直接应用该分 析方法会产生边缘效应, 所以一些针对该方法的改 进工作也主要集中在边缘效应的修正 (Donnelly, 1978; Füldner, 1995)方面。戴小华和余世孝(2003)借 助地理信息系统软件的功能支持, 采用 Füldner (1995)边缘修正后的最近邻体公式, 对规则样地和 不规则样地中的种群分布格局进行了研究, 结果表 明用最近邻体法分析这两种类型的样地内种群分布 格局都非常有效。但该方法只能分析特定尺度下的 格局特征, 因此一直被归入单尺度格局分析方法这 一类中 ( Perry et al .,2002)。

种群分布格局类型的确定与空间尺度相关
(Greig-Smith, 1983; Stewart \& Rose, 1990; 张金屯, 1998），一个种在小尺度下可能呈现聚集分布, 而在 大尺度下有可能为随机分布或均匀分布, 即种群空 间分布格局存在着尺度依赖性。但最近邻体分析只 能检验个体分布的随机性, 不能确定种群分布格局 随尺度而变化的情况 (Richards \& Williamson, 1975; 徐化成等, 1994; 王峥峰等, 1998)。因此, 本文提出 扩展最近邻体分析法, 并结合实例研究了广东省黑 石顶森林群落中 5 个树木种群的多尺度分布格局特 征, 旨在探讨如何改进传统单尺度分析方法以进行 多尺度格局分析的途径, 为研究格局与尺度的关系 提供新的思路。

\section{1 最近邻体分析的基本理论}

最近邻体分析最早由 Clark 和 Evans (1954) 提 出, 其理论是通过比较样地内种群的分布与假设的 随机分布之间的差异, 作出种群分布类型的判断。 该分析法以比值 $r_{\mathrm{A}} / r_{\mathrm{E}}$ 为最终结果, 当该比值等于 1 时为随机分布, 小于 1 时为聚集分布, 大于 1 时为均 匀分布。其中, $r_{\mathrm{A}}$ 为样地内各个体与其最近邻体间 距离的平均值, $r_{\mathrm{E}}$ 为相同环境中随机状态下距离的 平均值。Donnelly ( 1978 ) 对其进行了边缘校正, Füldner(1995)把样地周长对边缘效应的影响也考虑 进来, 对其作进一步的边缘校正。经校正后的 Clark-Evans 指数的计算公式 (Kint et al.,2000) 为:

$$
C E=r_{\mathrm{A}} / r_{\mathrm{E}}=\left(\frac{1}{N} \sum_{i=1}^{N} r_{i}\right) /(0.5 \sqrt{A / N}+0.0514
$$

$\left.P / N+0.041 P / N^{3 / 2}\right)$

式中: $C E$ 为 Clark-Evans 指数, $N$ 为样地内个体总 数, $A$ 为样地面积 $\left(\mathrm{m}^{2}\right), P$ 为样地周长 $(\mathrm{m}), r_{i}$ 为第 $i$ 个个体与其最近邻体间的距离 $(\mathrm{m})$ 。 
实际 $C E$ 指数值偏离 1 的显著性用标准正态分 布作检验( Clark \& Evans, 1954; Kint et al ., 2000), 其 计算式为:

$$
\begin{aligned}
& u=\left(r_{A}-r_{E}\right) / \sigma \\
& \sigma=0.26136 / \sqrt{\rho N}=0.26136 / \sqrt{N^{2} / A}
\end{aligned}
$$

式中: $\sigma$ 为标准差, $\rho=N / A$ 为样地内个体密度。

\section{2 扩展最近邻体分析}

扩展最近邻体分析 (Extended nearest neighbor analysis, ENNA) 是在传统最近邻体分析方法的基础 上, 增加一个距离尺度参数 $d(\mathrm{~m})$, 并使 Clark-Evans 指数 $C E$ 成为它的函数, 即 $C E(d)=f(d)$ 。这样, 随距离尺度 $d$ 的改变就可得到不同的 $C E(d)$ 指数 值, 从而实现种群多尺度分布格局的检验。

由公式(1) 可得计算 $C E$ 所需的全部条件为 $A$ 、 $N 、 P$ 和 $r_{i}$ 等 4 个变量, 其中具有决定意义的条件参 数为样地面积 $A$ 。因为对矩形或方形这类特定形状 的样地而言, 若其面积 $A$ 为已知, 则其周长 $P$ 、样地 内的总个体数 $N$ 和第 $i$ 个个体与其最近邻体间的距 离 $r_{i}$ 均可由计算机程序间接获得, 比如通过 $\mathrm{Ar}$ cView GIS 软件的最近邻体分析模块获得(戴小华和 余世孝,2003)。因此面积参数 $A$ 实际上暗含了一 个检验分布格局的特定距离尺度 $d$, 如矩形样地的 边长。使此距离尺度 $d$ 参数化, 即可借用传统的最 近邻体分析法作种群分布格局的多尺度分析。

具体地, 增加一个距离尺度参数 $d$, 由它来控制 面积 $A\left(\mathrm{~m}^{2}\right)$, 进而控制其它各参数, 即新的条件由 $d 、 A 、 N 、 P$ 和 $r_{i}$ 等 5 个变量组成。据此, 对公式 (1) 进行距离尺度 $d$ 参数化处理, 有:

$$
C E(d)=r_{d \mathrm{~A}} / r_{d \mathrm{E}}=\left(\frac{1}{N_{d}} \sum_{i=1}^{N_{d}} r_{d i}\right) /\left(0.5 \sqrt{A_{d} / N_{d}}+\right.
$$

$\left.0.0514 P_{d} / N_{d}+0.041 P_{d} / N_{d}{ }^{3 / 2}\right)$

相应地,正态分布检验计算式变为:

$u(d)=\left(r_{d \mathrm{~A}}-r_{d \mathrm{E}}\right) / \sigma_{d}$

$\sigma_{d}=0.26136 / \sqrt{\rho_{d} N_{d}}=0.26136 / \sqrt{N_{d}^{2} / A_{d}}$ （6）

公式(4)、(5)、(6)中各参数 $r_{d \mathrm{~A}} 、 r_{d \mathrm{E}} 、 N_{d} 、 r_{d \mathrm{i}} 、 A_{d} 、$ $P_{d} 、 \sigma_{d}$ 的意义分别为: 在距离尺度 $d(\mathrm{~m})$ 范围内, 样 地内各个体与其最近邻体间距离的平均值 $(\mathrm{m})$ 、相 同环境中随机状态下距离的平均值 $(\mathrm{m})$ 、样地内个 体总数、第 $i$ 个个体与其最近邻体间的距离 $(\mathrm{m})$ 、样 地面积 $\left(\mathrm{m}^{2}\right)$ 、样地周长 $(\mathrm{m})$ 、标准差。

根据公式 (4), 如果 $C E(d)$ 小于 1 则认为种群 为聚集分布, 等于 1 时为随机分布, 大于 1 时为均匀
分布。最终结果的判断还要通过 (5) 式的显著性检 验。利用公式 (4) 还可得到 $C E(d)$ 与 $d$ 的关系图, 用于图示分析依赖于距离尺度 $d$ 的种群空间分布 格局。

\section{3 研究实例}

\section{1 样地调查与数据预处理}

在广东省黑石顶自然保护区内选择样地作扩展 最近邻体分析的实例研究。有关黑石顶自然保护区 概况描述及其植被分类方案参见文献 (余世孝等, 2000)。

在保护区石门塘西北的架桥坑 (地理坐标为 $23^{\circ} 26^{\prime} \mathrm{N}, 111^{\circ} 53^{\prime} \mathrm{E}$ ) 设置一块面积为 $64 \mathrm{~m} \times 64 \mathrm{~m}$ 的样 地, 对样地内胸径 $1 \mathrm{~cm}$ 以上的乔灌木物种进行每木 调查, 调查内容包括种名、胸径、高度、冠幅和个体在 样地中的坐标位置。该群落为黑石顶自然保护区分 布最广的混交林类型一以马尾松为优势的针阔叶混 交林, 马尾松属衰退种群, 其在群落中的地位与阔叶 树相近, 其林相与阔叶林很相似(余世孝等, 2000)。

\section{2 点分布图}

采用点分布图来直观显示树木个体在样地内的 分布格局, 对种群分布格局进行可视化的定性分析 是种群分布格局研究中常用的作法 (Haase, 1995; Kint et al., 2000; Perry et al., 2002; 张金屯, 1998; 汤 孟平等,2003; 戴小华和余世孝,2003)。点分布图是 指把植物个体当作空间中的点, 以其在样地所处坐 标来表示其空间位置的一种散点图。它可直观地表 达种群在样地内的空间分布状态, 是定量研究的辅 助手段, 其所用数据是扩展最近邻体分析的来源。

群落样地共有树木 2288 株, 其中, 群落上层乔 木马尾松( Pinus massoniana)172 株, 阔叶树主要有黄 牛奶树 (Symplocos laurina) 102 株、水栗 (Castanopsis nigrescens) 118 株、鼠刺( Itea chinensis) 183 株等, 灌木 层桃金娘 (Rhodomyrtus tomentosa) 346 株。上述 5 个 种群的点分布图见图 1。由点分布图, 在 $64 \mathrm{~m} \times 64$ $m$ 的尺度范围内, 马尾松 (图 1a) 和水栗 (图 1c) 两个 种群有随机分布趋势, 而鼠刺(图 1d)有明显的聚集 分布趋势, 其它两个种群无明显分布趋势。

\section{3 最近邻体分析模块}

用于分析的程序是在 ArcView 最近邻体分析扩 展模块 Jeff Jenness's Nearest Features V.3.6d 的基础 上, 利用其 Avenue 语言进行二次开发所得。最近邻 体分析的基本过程是求得测区内或其随机样本中全 部个体与其最近邻体间的距离, 用平均最近邻体距 

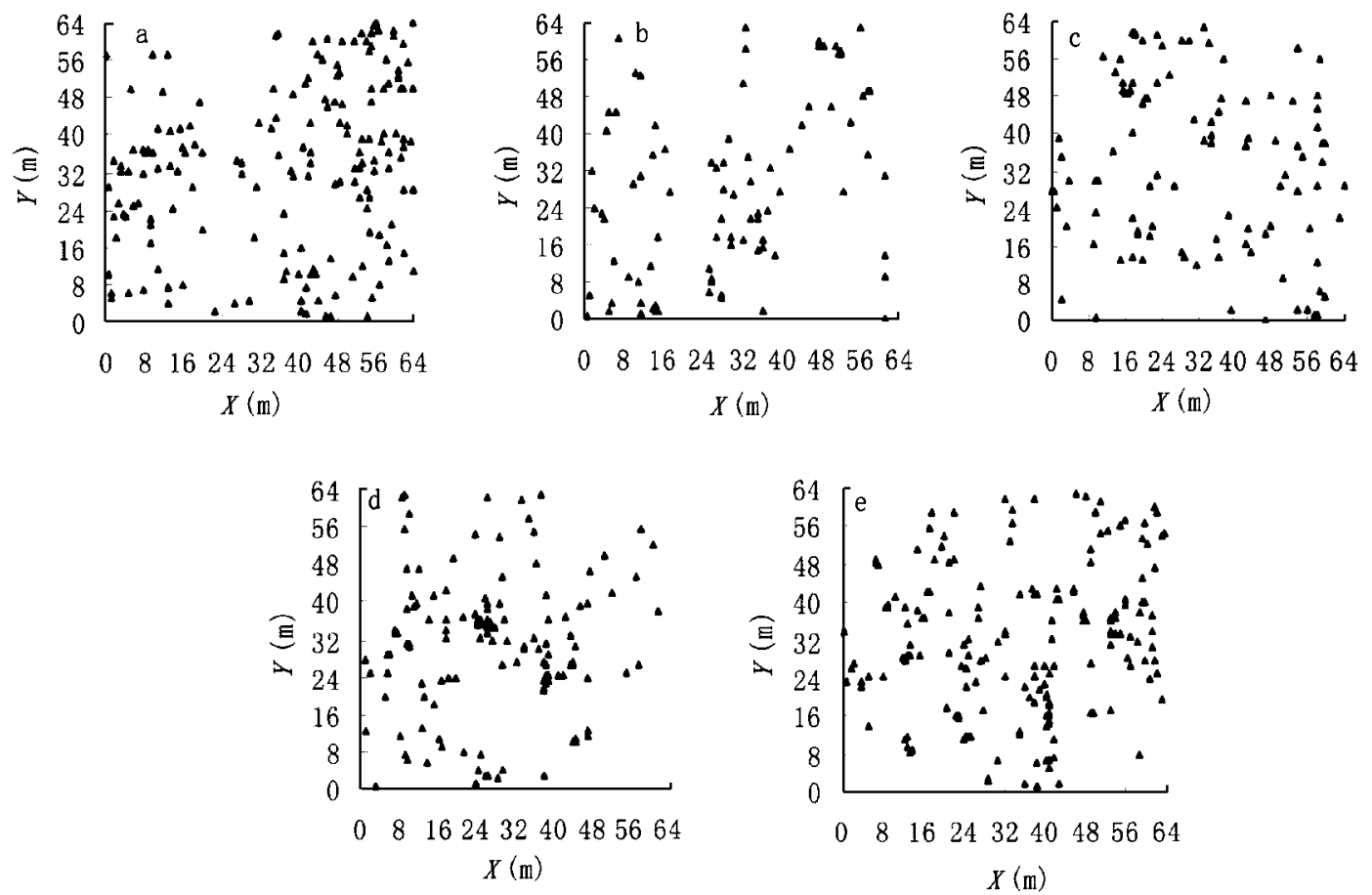

图 15 个树木种群的点分布图

Fig. 1 Mapped points pattern of five tree populations

a. 马尾松 Pinus massoniana b. 黄牛奶树 Symplocos laurina c. 水栗 Castanopsis nigrescens d. 鼠刺 Itea chinensis e. 桃金娘 Rhodomyrtus tomentosa

离与其为随机分布时的期望平均距离比较, 根据比 率偏离随机性的统计显著性判定种群分布格局的类 型(Clark \& Evans, 1954)。尺度变换方法仿分形理论 中沙盒维数的计算方法 (董连科, 1991)。整个运算 与分析过程由程序自动完成。

\section{4 结果与分析}

分布格局曲线图以图示化的方式反映 $C E(d)$ 指数与距离尺度 $d$ 之间的关系, 描述种群分布格局 随距离尺度变化而变化的走势。结果表明(图 2), 在黑石顶针阔叶混交林群落中, 马尾松显著表现为 随机分布, 鼠刺种群显著表现为聚集分布, 黄牛奶 树、水栗、桃金娘等 3 个种群的分布格局都表现出不 同程度的尺度相关性。随距离尺度的增加, 这 4 个 种群的分布格局以聚集或随机分布为主要类型。

鼠刺(图 2d) 在各个尺度范围内 $C E(d)$ 指数值 均小于 1 , 倾向于聚集分布。显著性检验的结果也 表明, 该种群在 $20 \mathrm{~m}$ 以上尺度范围内均为聚集分 布。

马尾松(图 2a)、黄牛奶树(图 2b)、水栗(图 2c)、 桃金娘(图 2e) 等 4 个种群的分布格局都表现出不同 程度的尺度相关性。它们的 $C E(d)$ 曲线在 $0 \sim 64 \mathrm{~m}$
整个距离尺度内围绕指数值 1 上下波动; 且在 $0 \sim 16$ $\mathrm{m}$ 尺度范围内, $C E(d)$ 和 $u(d)$ 曲线的波动幅度均较 大, 在此尺度范围内进行分布格局检验, 其结果之间 的差异很大。表明在进行种群格局多尺度分析时可 能存在一个最小尺度, 多尺度分析宜在大于该尺度 范围内进行。本研究中 $16 \mathrm{~m}$ 可能是适合进行多尺 度分析的最小尺度。

在距离尺度 $20 \sim 64 \mathrm{~m}$ 范围内, 马尾松（图 2a） $C E(d)$ 指数值在 1 附近以较小的幅度平稳波动, 说 明该种群有随机分布的趋势, 分布格局检验的结果 也为随机分布, 其点分布图 (图 1a) 也直观地反映这 一点; 此尺度范围内水栗种群 (图 1c, 图 2c) 的情况 与之相似, 但该种群在 $40 \sim 44 \mathrm{~m}$ 尺度范围内出现聚 集分布格局。

在距离尺度 36 48 m 范围内, 黄牛奶树 (图 2b) 的 $C E(d)$ 指数值出现较大幅度的上升, 种群呈均匀 分布; 但随尺度进一步增大, $C E(d)$ 指数值不断下 降, 种群经由随机分布变为聚集分布。桃金娘种群 (图 1e, 图 2e) 在 4 12 m、20 28 m、44 52 m 等尺 度范围内出现均匀分布格局, 而在其它尺度范围内 均表现为随机分布。 


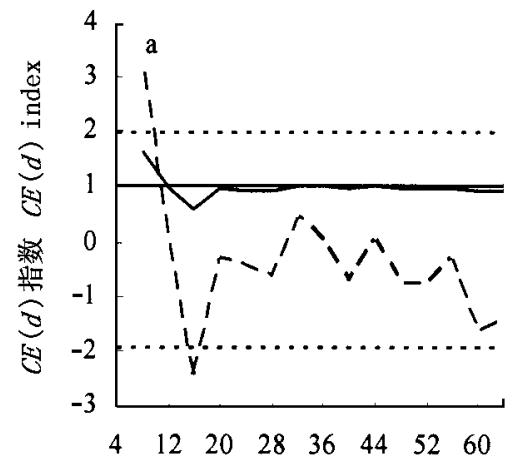

距离尺度 Distance scale (m)

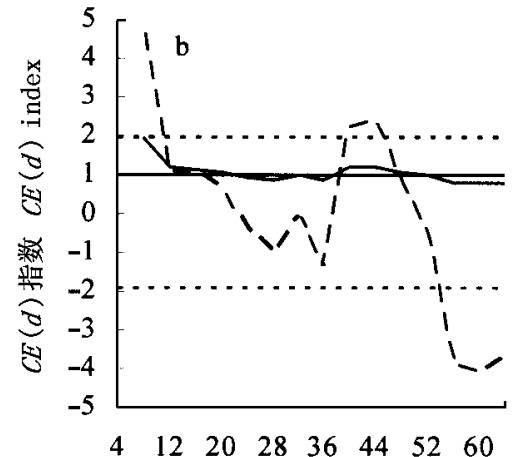

距离尺度 Distance scale (m)

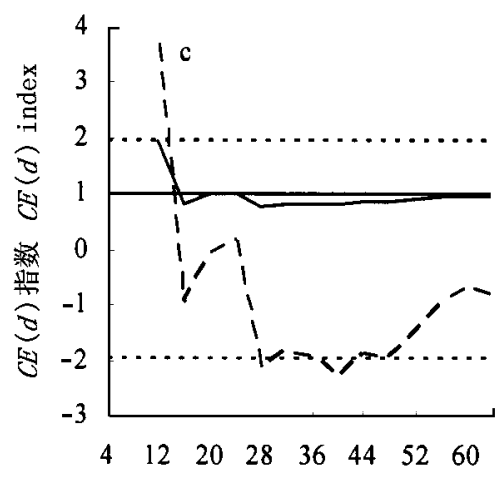

距离尺度 Distance scale (m)

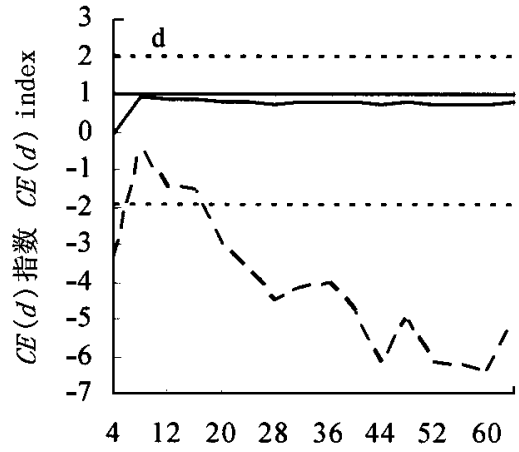

距离尺度 Distance scale (In)

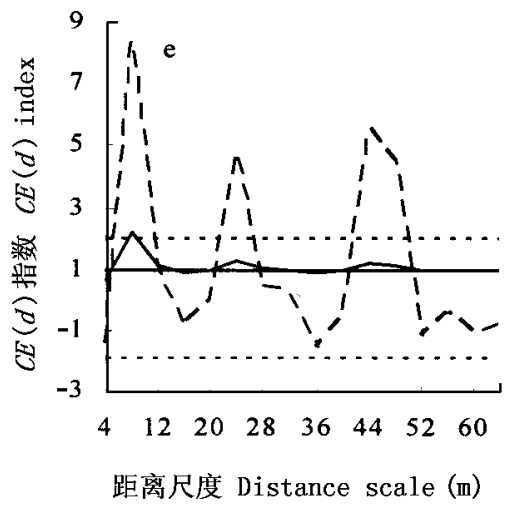

$\longrightarrow C E(d)$ 曲线 $C E(d)$ curve - - $u(d)$ 曲线 $u(d)$ curve

临界值线 Critical value lines

图 25 个树木种群的分布格局

Fig.2 Spatial pattern of five tree populations

a. 马尾松 Pinus massoniana b. 黄牛奶树 Symplocos laurina $\quad$ c. 水栗 Castanopsis nigrescens d. 鼠刺 Itea chinensis e. 桃金娘 Rhodomyrtus tomentosa

鼠刺属耐荫性树种, 该种群容易发展为常绿阔 叶林的伴生种或建群种, 其聚集分布格局一定程度 上预示其所处群落正向常绿阔叶林发生进展演替。 而马尾松、黄牛奶树、水栗、桃金娘等 4 种群分布格 局的尺度相关性, 以及在距离尺度 $52 \mathrm{~m}$ 以上,马尾 松、水栗、桃金娘等 3 种群均表现为随机分布格局的 结果表明, 它们在群落中所处地位差异不大, 反映该 针阔叶混交林正处于针叶树与其它阔叶树相对平衡 的过渡阶段, 群落演替趋势是针叶树种 (主要是马尾 松)进一步衰退( 丁圣彦和宋永昌, 1998)，阔叶树种 进一步发展, 整个群落将发展成为以阔叶树为主的 群落类型(周先叶等, 1999; 各启杰等, 2000)。

\section{4 讨论与结论}

\section{1 关于 5 个种群的多尺度分布格局}

关于种群空间分布格局的尺度依赖性已有论述 (Greig-Smith, 1983; Stewart \& Rose, 1990; 张金屯,
1998)。本研究的结果(图 2) 也表明, 随距离尺度 $d$ 的改变, 各种群的 $C E(d)$ 指数曲线均出现不同程度 的波动, 意味着种群分布格局存在尺度依赖性。其 可能原因是: 1)种群个体数的变化。尺度的改变意 味着研究区域的改变, 因而其内种群个体数也随之 改变, 则由公式(5)可知, 格局分析的结果必然改变。 2)种群所处生境条件等因素的变化。生态学系统的 空间异质性是生物系统的主要属性之一(李哈滨等, 1998), 而它是尺度的函数, 即其变异依赖于时空尺 度 ( Li \& Reynolds, 1995)。因此, 处于异质性空间中 的种群其分布格局也会表现出相应的变化。3)种 内、种间关系的变化。最近邻体分析方法的一个隐 含假设就是, 植物种群的相互作用主要发生在其最 邻近的个体之间。当这种作用发生在同种之间时, 即发生自疏现象; 发生在异种之间时,即发生他疏现 象。已有研究表明 (侯向阳和韩进轩, 1997), 在时间 尺度上, 林木的随机自疏是种群格局动态变化的重 
要原因。本研究表明, 随空间尺度的改变, 自疏和他 疏现象的作用范围也会相应改变, 小尺度上形成的 格局会在较大尺度上进行复合, 进而表现为种群格 局的尺度变化。

广东省黑石顶自然保护区地处南亚热带, 该区 森林群落普遍存在由马尾松林向常绿阔叶林演变的 趋势( 周先叶等, 1999; 各启杰等, 2000), 马尾松既是 先锋种, 又是消退种 (丁圣彦和宋永昌, 1998)。图 2 表明, 在 $16 \sim 64 \mathrm{~m}$ 较宽的尺度范围内, 马尾松种群 均呈随机分布格局, 种群个体的随机死亡可能是其 主要原因。因所研究群落为处于森林群落演替中期 的针阔叶混交林阶段, 水栗、鼠刺等其它阔叶树种已 逐渐占据群落上层, 并将发展为群落中的优势种, 而 马尾松种群则处于消退状态。根据丁圣彦和宋永昌 (1998) 的研究结果, 一方面, 因群落内光照强度过 低, 使马尾松种群更新苗处于光补偿点之下, 难以生 长; 另一方面, 群落内耐荫树种的抑制使马尾松成树 的物质合成能力逐渐减弱, 物质分解能力逐渐加强, 以致其在演替过程中逐渐消失。由自疏或他疏引起 的随机死亡将导致种群格局由聚集分布向均匀分布 偏移 (Antonovics \& Levin, 1980; Peterson \& Squiers, 1995 ), 而随机分布则是这种偏移过程的中间状态。

\section{2 关于扩展最近邻体方法}

生态学中的空间尺度是指所研究生态系统的面 积大小, 它是对所研究对象的细节了解程度的标志 (肖笃宁等, 1997)。目前对尺度的研究已经引起了 广泛重视，但还不够成熟(吕一河和傅伯杰,2001)。

尺度与格局的概念必定是相互交织在一起的 (陈玉福和董鸣, 2003)。格局在不同尺度上的类型 及其属性一般不同, 因而表现出格局的尺度依赖性 或尺度变异性。只有关注尺度效应, 才能得出切实 结论; 对每一尺度上植被格局的个性特征详细考察 以获得直观的结果被认为是解决尺度依赖问题的重 要途径(马克明和祖元刚, 2000)。

以广东省黑石顶自然保护区针阔叶混交林中的 马尾松、黄牛奶树、水栗、鼠刺和桃金娘等 5 个代表 性种群为实例的研究表明, 5 个种群均表现出不同 程度的尺度相关性。应用本文提出的扩展最近邻体 分析方法 ENNA 能够检测出种群空间分布格局的尺 度依赖性, 获得关于种群空间分布格局的多尺度信 息, 其分析结果具有明显的生态学意义, 因而, 该方 法是进行种群多尺度空间格局分析的有效方法。进 一步还可采用本文的扩展思路对其它隐含距离信息 的传统分析方法作类似的改进, 使之获得多尺度分
析功能, 以进行生态学问题的多尺度研究。因而, 本 文研究的思路还具有一定的方法论意义。

在地理信息系统软件 ArcView 的支持下, 扩展 最近邻体分析方法还可用于对多个种群的空间分布 格局进行多尺度检验, 其结果可用于发现具有相似 分布格局的种组, 即格局种组, 以进行其它相关生态 学研究; 或寻找相同种群其分布格局存在差异的原 因, 如生长阶段的差异、生境异质性等。因此, 扩展 最近邻体分析方法具有较好的生态学应用前景。

\section{参 考 文 献}

Antonovics J, Levin DA (1980) . The ecological and genetic consequences of density-dependent regulation in plants. Annual Review of Ecology and Systematics, 11, $411-452$.

Chen YF (陈玉福), Dong M (董鸣) (2003). Spatial heterogeneity in ecological systems. Acta Ecologica Sinica (生态学报), 23, 346 - 352. (in Chinese with English abstract)

Clark PJ, Evans FC (1954). Distance to nearest neighbour as a measure of spatial relationships in populations. Ecology, 35, 445 -453 .

Dai XH (戴小华), Yu SX (余世孝) (2003). Analysis of population distribution pattern based on GIS technique. Acta Scientiarum Naturalium Universitatis Sunyatseni (中山大学学报 (自 然科学版) ), 42, 75-78. (in Chinese with English abstract)

Ding SY (丁圣彦), Song YC (宋永昌) (1998). Declining causes of Pinus massoniana in the process of succession of evergreen broad-leaved forest. Acta Botanica Sinica (植物学报), 40, 755 - 760. (in Chinese with English abstract)

Dong LK (董连科) (1991). Fractal Theory and Its Applications. Liaoning Science and Technology Publishing House, Shenyang. (in Chinese)

Donnelly KP (1978). Simulations to determine the variance and edge effect of total nearest-neighbour distance. In: Hodder I ed. Simulation Studies in Archaeology. Cambridge University Press, Cambridge, 91 - 95.

Füldner K (1995). Zur Structurbeschreibung in Mischbeständen. Forstarchiv, 66, $235-240$.

Greig-Smith P (1983). Quantitative Plant Ecology 3rd edn. Blackwell Scientific Publications, London.

Haase P (1995). Spatial pattern analysis in ecology based on Ripley's $K$-function: introduction and methods of edge correction. Journal of Vegetation Science, 6, 575-582.

Hou XY (侯向阳), Han JX (韩进轩) (1997). Simulation analysis of spatial patterns of main species in the Korean-pine broadleaved forest in Changbai Mountain. Acta Phytoecologica Sinica (植物生态学报), 21, 242-249. (in Chinese with English abstract)

Kint V, Lust N, Ferris R, Olsthoorn AFM (2000). Quantification of forest stand structure applied to Scots pine (Pinus sylvestris L.) forests. In: Mason B ed. Silviculture and Biodiversity of Scots Pine Forests in Europe. Proceeding of the Final Meeting of a Concerted Action, June 1999, Valsain, Spain. Investigación Agraria: Sistemas y Recursos Forestales, Fuera de Serie, 1, 147 164. 
Li H, Reynolds JF (1995) . On definition and quantification of heterogeneity. Oikos, 73, 280-284.

Li HB (李哈滨), Wang ZQ (王政权), Wang QC (王庆成) (1998). Theory and methodology of spatial heterogeneity quantification. Chinese Journal of Applied Ecology (应用生态学 报), 9, 651-657. (in Chinese with English abstract)

Lï YH (吕一河), Fu BJ (傅伯杰) (2001). Ecological scale and scaling. Acta Ecologica Sinica (生态学报), 21, 2096-2105. (in Chinese with English abstract)

Ma KM (马克明), Zu YG (祖元刚) (2000). Fractal properties of vegetation patterns. Acta Phytoecologica Sinica (植物生态学 报), 24, 111 - 117. (in Chinese with English abstract)

Moeur M (1993). Characterizing spatial patterns of trees using stem-mapped data. Forest Science, 39, 756 - 775.

Perry JN, Liebhold AM, Rosenberg MS, Dungan J, Miriti M, Jakomulska A, Citron-Pousty S (2002). Illustrations and guidelines for selecting statistical methods for quantifying spatial pattern in ecological data. Ecography, 25, 578-600.

Peterson JC, Squiers ER (1995). An unexpected change in spatial pattern across 10 years in an aspen-white-pine forest. Journal of Ecology, 83, 847 - 855.

Richards P, Williamson GB (1975). Treefalls and patterns of understory species in a wet lowland tropical forest. Ecology, 56, 1226 - 1229 .

Stewart GH, Rose AB (1990). The significance of life history strategies in the developmental history of mixed beech forests, New Zewland. Vegetatio, 87, $101-114$.

Tang MP (汤孟平), Tang SZ (唐守正), Lei XD (雷相东), Zhang HR (张会儒), Hong LX (洪玲霞), Feng YM (冯益 民) (2003). Edge correction of Ripley's $K(d)$ function on population spatial pattern analysis. Acta Ecologica Sinica (生态 学报), 23, 1533-1538. (in Chinese with English abstract) Wang ZF (王峥峰), An SQ (安树青), Zhu XL (朱学雷),
Campell DG, Yang XB (杨小波) (1998). Distribution pattern of tree populations in tropical forest and comparison of its study methods. Chinese Journal of Applied Ecology (应用生态学报), 9, 575 - 580. (in Chinese with English abstract)

Xiao DN (肖笃宁), Bu RC (布仁仓), Li XZ (李秀珍) (1997). Spatial ecology and landscape heterogeneity. Acta Ecologica Sinica (生态学报), 17, 453 - 461. (in Chinese with English abstract)

Xu HC (徐化成), Fan ZF (范兆飞), Wang S (王胜) (1994). A study in spatial patterns of trees in virgin Larix gmelini forest. Acta Ecologica Sinica (生态学报), 14, 155 - 160. (in Chinese with English abstract)

Yu SX (余世孝), Li Y (李勇), Wang YF (王永繁), Zhou CF (周灿芳) (2000). The vegetation classification and its digitized map of Heishiding Nature Reserve, Guangdong I. The distribution of the vegetation type and formation. Acta Scientiarum Naturalium Universitatis Sunyatseni (中山大学学报 (自然科学 版) ), 39,61-66. (in Chinese with English abstract)

Zan QJ (处启杰), Li MG (李鸣光), Wang BS (王伯荪), Zhou $\mathrm{XY}$ (周先叶) (2000). Dynamics of community structure in successional process of needle and broad-leaved mixed forest in Heishiding of Guangdong. Chinese Journal of Applied Ecology (应用生态学报), 11, 1-4. (in Chinese with English abstract)

Zhang JT (张金屯) (1998). Analysis of spatial point pattern for plant species. Acta Phytoecologica Sinica (植物生态学报), 22, 344 - 349. (in Chinese with English abstract)

Zhou XY (周先叶), Wang BS (王伯荪), Li MG (李鸣光), Zan QJ (处启杰) (1999). The community dynamics of the forest secondary succession in Heishiding Natural Reserve of Guangdong province. Acta Botanica Sinica (植物学报), 41, 877 886. (in Chinese with English abstract)

责任编委: 张大勇 责任编辑: 刘丽娟 